\title{
Comparison of CFD with Reservoir Routing Model Predictions for Stormwater Ponds
}

\section{Bernardo C. Trindade and Jose G. Vasconcelos}

The hydraulic design of stormwater detention ponds is usually directed towards outflow peak reduction. It is generally calculated with the reservoir routing approach, which is essentially a discrete solution for the continuity equation at such ponds. The reservoir routing approach has been successful in predicting this outflow peak attenuation, and is implemented in various computational models such as SWMM 5 (USEPA, 2009). However, the reservoir routing approach does not provide information on the velocity fields that are expected within the pond. This parameter could be useful in assessing the location and extent of dead zones, as well as regions in which velocities may be outside a desired range. Such velocity fields can be obtained with the use of more complex computational fluid dynamics (CFD) models, but such models usually have steep learning curves and are also computationally intensive applications that may be expensive to perform for simpler reservoir systems. This chapter presents the result of a capstone project in which a comparative study of the flow predictions yielded by the reservoir routing approach and those of a two dimensional CFD model. The CFD model has a very user friendly interface, and is applied to two hypothetical detention ponds, one of them being a simplified version of a pond in São Paulo, Brazil. The shape and the peak discharge in the outflow hydrographs obtained by the CFD model were compared, and the locations of high and low velocity zones for each simulation condition were also determined with the model. The results indicate that the peak discharges in both methods agreed very well, but there are some discrepancies in the predicted shapes of the hydrographs after the peak flows. Results presented in this chapter show

Trindade, B.C. and J. Vasconcelos. 2011. "Comparison of CFD with Reservoir Routing Model Predictions for Stormwater Ponds." Journal of Water Management Modeling R241-05. doi: 10.14796/JWMM.R241-05. (C) CHI 2011 www.chijournal.org ISSN: 2292-6062 (Formerly in Cognitive Modeling of Urban Water Systems. ISBN: 978-0-9808853-4-7) 
that velocity profiles may be useful in order to avoid configurations that will lead to scour and undesirable sediment deposition.

\subsection{Introduction}

The reservoir routing approach is possibly the most common method used for the analysis of detention ponds, which are frequently used in stormwater drainage systems. Experience has shown that this method is effective for predicting the peak outflow attenuation in such reservoirs, and it is implemented in various computational models such as SWMM 5 (USEPA, 2009). It uses the principle of continuity to solve the flows and assumes a flat water surface inside the detention pond during the entire computation. However, the reservoir routing approach provides no information regarding the velocity fields within the reservoir. This lack of information makes it difficult to assess where high velocity zones, linked to scouring processes, would occur. Another issue concerns zones of short circuit flow and low velocities. Knowing the location of these zones would help to predict maintenance needs and provide information to determine the quality of the outflow water.

An alternative to the reservoir routing approach is the use of more sophisticated numerical techniques based on CFD. For instance, in an application involving abrupt changes in river water levels, Miyaoka and Kawahara (2008) used a CFD model to obtain an outflow discharge that minimizes the draining time of a given detention pond, while still maintaining the water level at a downstream point in the river below a predetermined value. Their model was based on the shallow water equations (SWE) implemented with the finite element model, considering a moving boundary and optimal efficiency theory. With the numerical model, an optimal control velocity for a small detention pond was obtained and the water elevation in the Tsurumi River was verified to be almost coincident with the target value, increasing its efficiency in avoiding floods in part of the city of Tokyo.

Yet, as pointed by Qiu and Fang (2009), widespread application of CFD models is still unlikely to take place in the near future. The authors performed several physical and numerical experiments in order to demonstrate some challenges for students and professional practitioners regarding CFD modeling. They concluded that, although such modeling can return results which agree with those of the experimental data, to achieve the correct results requires of the user a deep knowledge of hydraulic theory in order to properly set the software parameters and boundary conditions. Besides, CFD models are usually expensive, the training time is extensive and the learning curve is steep. This may help to explain why such CFD models are seldom used for detention pond analysis. 


\subsection{Objectives}

This study presents the basis of a new tool for the analysis of detention ponds based on a CFD model and performs a comparison with the traditional reservoir routing approach. The proposed CFD model assumes the flow inside the detention ponds to be mainly depth-averaged, bidimensional and transient, which allows the application of SWE. Using these equations, it is possible to compute velocity fields and variations of the water surface over time.

The velocities profile calculation, which is performed by the proposed model, is the first step towards the development of a model capable of predicting sedimentation and scour zones in detention ponds. With further research, sedimentation and shear stresses models could be coupled with the velocities profiles calculation, making this CFD model a more complete one for detention pond analysis, yet simple to be learned and applied. In order to be as simple to operate as possible, with a user friendly graphical user interface. Unlike generic CFD solvers able to solve several different conditions, only the basic features needed to simulate the flow in detention ponds were included in the proposed model. This reduces the learning time which would be required to use all model capabilities.

Besides presenting the CFD approach to the problem, a comparison between the presented CFD model and the reservoir routing approach is presented in terms of the peak outflow value and hydrographs for two hypothetical detention ponds.

\subsection{Governing Equations and Numerical Methods}

\subsubsection{Classical Reservoir Routing Approach}

This method attempts to find an accurate numerical solution to the continuity equation:

$$
I-Q=\frac{d V}{d t}
$$

where:

$$
\begin{aligned}
I & =\text { inflow discharge } \\
Q & =\text { outflow discharge } \\
V & =\text { volume of water stored, and } \\
t & =\text { time. }
\end{aligned}
$$


To solve this ordinary differential equation, the free surface is assumed flat during the entire simulation and the relation between the head and the discharge through the outlet structure is predetermined. According to Paine and Akan (2001) Equation 5.1 in finite difference form becomes:

$$
Q_{t+1}+\frac{2 \cdot S_{t+1}}{\Delta t}=I_{t+1}+I_{t}-Q_{t}+\frac{2 \cdot S_{t}}{\Delta t}
$$

where $S_{t}$ is the accumulated volume of water in the time step and $\Delta t$ the chosen time step.

Since there are two unknowns $\left(Q_{t+l}\right.$ and $\left.S_{t+1}\right)$ another equation is needed to solve the problem. For this, relations head vs discharge and head vs storage are used in order to obtain a discharge vs storage expression in the form $Q=f(S)$. The head vs discharge relation is normally the orifice/weir equation, and the head vs storage relation, according to Paine and Akan (2001), is:

$$
S=b \cdot h^{c}
$$

where:

$b$ and $c$ are parameters depending on the pond's geometry and topography.

Since $S$ can be found using Equation 5.3, the left hand side of Equation 5.2 becomes specified and the updated outlet discharge $Q_{t+1}$ and volume $S_{t+1}$ can be determined. This discharge is hereafter defined as reservoir routing discharge.

$$
Q=f\left(Q+\frac{2 \cdot S}{\Delta t}\right)
$$

\subsubsection{Flow Solution Using the SWE}

Developed as a simplified version of the Euler equation, the SWE assumes that the following assumptions can be applied to the free surface flow (Toro 2001):

1. The vertical acceleration can be neglected, so the flow can be considered to be depth averaged two-dimensional. This assumption usually applies to flows with the two horizontal dimensions much greater than the vertical dimension, like the flow in detention ponds, dam breaks and shallow beaches; and

2. The fluid (water) can be considered nearly incompressible. For free surface water flows with low depth this is a valid as- 
sumption because the pressures are too small and the Young modulus of water is rather big.

Applying those hypotheses to the Euler equations yields the SWE:

$$
\left\{\begin{array}{c}
\frac{\partial h}{\partial t}+\frac{\partial(h u)}{\partial x}+\frac{\partial(h v)}{\partial y}=0 \\
\frac{\partial(h u)}{\partial t}+\frac{\partial}{\partial x}\left(u^{2} h+g \frac{h^{2}}{2}\right)+\frac{\partial}{\partial y}(u v h)=-g\left(S_{0 x}-S_{f x}\right) h \\
\frac{\partial(h v)}{\partial t}+\frac{\partial}{\partial x}(u v h)+\frac{\partial}{\partial y}\left(v^{2} h+g \frac{h^{2}}{2}\right)=-g\left(S_{0 y}-S_{f y}\right) h
\end{array}\right.
$$

where:

$$
\begin{aligned}
h & =\text { depth } \\
u & =\text { velocity in the horizontal direction } x, \\
v & =\text { velocity in the horizontal direction } y, \\
g & =\text { gravitational acceleration, } \\
S_{0} & =\text { topography of the bottom of the pond, and } \\
S_{f} & =\text { friction slope. }
\end{aligned}
$$

In the divergent vectorial form, Equation 5.4 is presented as:

$$
\begin{gathered}
\frac{\partial}{\partial t} \vec{U}+\frac{\partial}{\partial x} F(\vec{U})+\frac{\partial}{\partial y} G(\vec{U})=S(\vec{U}) \\
\vec{U}=\left(\begin{array}{c}
h \\
v h \\
v h
\end{array}\right) ; F(\vec{U})=\left(\begin{array}{c}
h u \\
u^{2} h+g \frac{h^{2}}{2} \\
u v h
\end{array}\right) ; G(\vec{U})=\left(\begin{array}{c}
h u \\
u v h \\
v^{2} h+g \frac{h^{2}}{2}
\end{array}\right) ; S(\vec{U})=\left(\begin{array}{c}
0 \\
-g\left(S_{o y}-S_{f y}\right) h \\
-g\left(S_{o y}-S_{f y}\right) h
\end{array}\right)
\end{gathered}
$$

with

$$
\begin{aligned}
\vec{U}=\text { conserved variables vector } \\
F(\vec{U}) \text { and } G(\vec{U})=\text { fluxes of conserved variables at the center of the } \\
\quad \text { cell, and } \\
S(\vec{U})=\text { source terms vector. }
\end{aligned}
$$

If shockwaves (hydraulic bores) occur in the reservoir, Equation 5.4 will not be able to represent them consistently because, mathematically, shockwaves represent spatial flow discontinuities. The spatial derivatives of Equations 5.4 and $5.5(\partial / \partial x$ and $\partial / \partial y)$ are not defined at a discontinuity, rendering those terms in Equation 5.4 undetermined (Vasconcelos, 2007). In order to avoid this problem, one alternative is to use the integral form of Equation 5.4. Integrating Equation 5.4 on the $x-y$ axis results in the integral form of the SWE: 


$$
\iint_{A} \frac{\partial}{\partial t} \vec{U} d x d y=-\iint_{A} \frac{\partial}{\partial x} F(\vec{U})+\frac{\partial}{\partial y} G(\vec{U}) d x d y+\iint_{A} S(\vec{U}) d x d y
$$

The finite volume method solution of the SWE is derived by applying the Gauss divergence theorem to the first term of the right side of Equation 5.7 and expressing the integrals discretely using the rectangular solution domain (Toro, 2001):

$$
\vec{U}_{i}^{n+1}=\vec{U}_{i}^{n}+\frac{\Delta t}{\Delta x}\left(\vec{F}_{i-\frac{1}{2}, j}^{n+\frac{1}{2}}-\vec{F}_{i+\frac{1}{2}, j}^{n+\frac{1}{2}}\right)+\frac{\Delta t}{\Delta y}\left(\vec{G}_{i, j-\frac{1}{2}}^{n+\frac{1}{2}}+\vec{G}_{i, j+\frac{1}{2}}^{n+\frac{1}{2}}\right)+\Delta t S_{i}^{n}
$$

where:

$$
\begin{aligned}
n & =\text { time step, } \\
i-1 / 2= & \text { interface between the calculation cells } i \text { and } i-1, \\
& \quad \text { making those fluxes the inter-cell fluxes, } \\
\Delta t= & \text { time step, and } \\
\Delta x \text { and } \Delta y= & \text { lengths of the cell } i \text { in the directions } x \text { and } y .
\end{aligned}
$$

The values of the inter-cell fluxes $F$ and $G$ (which are different from the $F$ and $G$ in Equation 5.6, which are the centered cell fluxes) are evaluated at each interface using numerical schemes. Equation 5.8 can solve the flow with or without discontinuities.

\subsubsection{Lax-Friedrichs Scheme}

This first order linear scheme is one of the simplest numerical schemes that can be used to calculate the inter-cell fluxes presented in Equation 5.7. Although it introduces significant numerical diffusion in the flow simulation (Vasconcelos 2007), it is fast to calculate and easy to implement in a computational code, which makes it a good choice for a preliminary study like this. Therefore, it was implemented in the proposed CFD model and its general formula in the bidimensional version is presented in Vasconcelos (2007) as:

$$
\left\{\begin{array}{c}
\vec{F}_{i+\frac{1}{2}, j}^{n+\frac{1}{2}}=\frac{1}{2}\left(\vec{F}_{i, j}^{n}+\vec{F}_{i+1, j}^{n}\right)+\frac{1}{4} \frac{\Delta t}{\Delta x}\left(\vec{U}_{i, j}^{n}+\vec{U}_{i+1, j}^{n}\right) \\
\vec{G}_{i, j+\frac{1}{2}}^{n+\frac{1}{2}}=\frac{1}{2}\left(\vec{G}_{i, j}^{n}+\vec{G}_{i, j+1}^{n}\right)+\frac{1}{4} \frac{\Delta t}{\Delta y}\left(\vec{U}_{i, j}^{n}+\vec{U}_{i, j+1}^{n}\right)
\end{array}\right.
$$

\subsection{Methodology}

For each example, the reservoir routing approach results were calculated with a spreadsheet. The SWE model, due to its complexity, was implemented through an Object Pascal program with a graphical user interface. 
The program includes a post-processor that is able to create an output report as a CSV (comma separated value) file, readable by spreadsheet programs. It is also integrated with the free software GNUPlot (Williams and Kelley, 2004), being able to export text files and to use them to generate 3-D surfaces and colored 2-D and isoline velocity fields maps.

Due to time constraints on an undergraduate capstone design project at the University of Brasilia (six months for literature review and six months for implementation), the model could not be calibrated and proper sedimentation and scour calculations could not be implemented because these require a level of work far beyond the scope of a capstone project. Yet, in order to illustrate future usage of this model to predict scour and sedimentation, very simplified criteria were adopted here to map regions more prone to such issues. In essence, it was proposed that:

- an average velocity for the total simulation time smaller than $5 \mathrm{~mm} / \mathrm{s}$ would make this point more prone to sedimentation; and

- a maximum velocity for the total simulation time greater than $2 \mathrm{~m} / \mathrm{s}$ would make this point more prone to scour if the bottom is not lined.

We acknowledge that the criteria presented above are very simple and not realistic. Sedimentation depends on several factors such as the relative density between fluid and sediment, fluid viscosity and sediment surfaces. Also, due to the time constraints, source terms were considered to be equal to zero as in Equation 5.5. Further development is required to properly implement source terms that would account for bottom topography, mild slopes $\left(\leq 5^{\circ}\right)$ and friction losses. Currently the model assumes that the ponds walls are vertical. For walls with slopes $>5^{\circ}$, the SWE cannot be used because the hypothesis that assumes non-vertical accelerations is inapplicable. In such a case, a 3D CFD code would be required. Hence to represent strong slopes proper boundary conditions would need to be developed.

For simplification, the cells are assumed to be always rectangular with uniform sizes $\Delta x$ and $\Delta y$. Non-perpendicular walls can be represented by series of rectangular cells disposed as pixels of a straight inclined line within the computational domain. As will be explained in section 5.4.2, the domain construction is created by drawing the domain contours with pixels representing a rectangular cell. Some of those pixels represent the inlet and outlet boundary conditions, while the majority represents solid boundaries.

\subsubsection{Boundary Conditions (BC) in the SWE Model}

\section{Wall (Reflexive Condition)}

This is the simplest boundary condition. The idea is to create a ghost cell at the boundaries and make the fluxes between the wall and the domain equal 
to zero by inverting the normal component of the velocity and setting the depth and the parallel velocity of the wall cell equal to that of the domain cell and keeping. The mathematical formulation of this boundary condition is showed by Toro (2001) for the one dimensional case as follows:

$$
\left\{\begin{array}{l}
h_{i+1}=h_{i} \\
u_{i+1}=-u_{i} \\
v_{i+1}=v_{i}
\end{array}\right.
$$

Figure 5.1 shows a typical wall. The black cells with a $p$ inside are the wall, the gray cells with the $\sim$ inside are the domain, and the blank cells are outside the domain. The same applies for the perpendicular direction with $j$ instead of $i$.

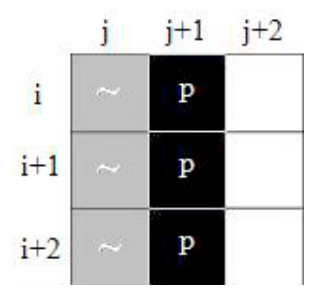

Figure 5.1 A typical wall.

$90^{\circ}$ Edges

This BC is very similar to the wall BC. Its layout is shown in Figure 5.2, which is schematized the same as Figure 5.1.

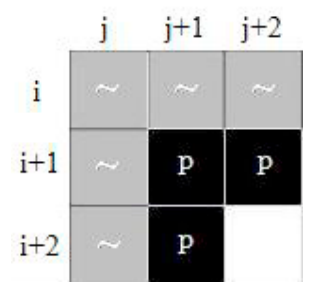

Figure 5.2 Typical $90^{\circ}$ edge.

This condition calculates the cell in the centre of Figure 5.2, which has two faces in contact with cells from the domain. This cell stores two values for each constraint ( $h, u$ and $v$ ), each value from one of the domain cells in contact with it, so that when the domain cells are being calculated each of them will get a different value of the constraints form the $90^{\circ}$ edge cell.

No boundary condition calculation is required for the dead corners because their values are not used by any other cell. 


\section{Inlet Hydrograph}

At inlets and outlets, the velocity is considered perpendicular to the wall. With this hypothesis, the unknowns at those cells are the depth and onedimensional velocity, perpendicular to the wall, with the parallel component being set to zero. Since there are two unknowns at those cells (velocity and depth) two equations are required to solve the flow at the inlet cells. Therefore, the inlet is calculated using the characteristic form of mass and momentum partial differential equations for free surface flow while it is enforced and a predetermined inlet hydrograph $Q_{\text {hydrograph }}(t)$, as shown in Sturm (2001). For this to proceed, the flow has to be subcritical or critical, since there are no characteristics lines coming back from the domain into the inlet BC for supercritical flows (Cunge et al., 1980). Hence, when the BC detects that the flow tends to become supercritical, the computational code enforces critical flow using the continuity principle and increasing the depth until Froude number reaches unity.

One other problem in using the method of characteristics for free surface flow is that the slope of the characteristics lines varies with time, which in turn makes the time step to vary during the calculations. To avoid this problem, a variation of the method of characteristics presented in Sturm (2001), the Hartree method, was used. In this method, fixed time and spatial intervals are specified and an interpolation between the spatial coordinates of the previous time steps is made for the characteristic line in order to find the right slope to link the previous and the present time step points. Those relations are used to build a single continuity equation that can be solved in terms of the velocity. The equations solved at this $\mathrm{BC}$ are:

$$
\begin{aligned}
& Q_{\text {in }}-\left(Q_{\text {acum }}+Q_{\text {out }}\right)=0 \\
& Q_{\text {in }}=f(t) \\
& Q_{\text {out }}=\left|V_{P}\right| \cdot h_{P} \cdot d x \\
& Q_{\text {acum }}=\frac{\left(h_{P}^{n}-h_{P}^{n-1}\right) \cdot d x \cdot d y}{d t}
\end{aligned}
$$

where:

$$
\begin{aligned}
Q_{\text {acum }} & =\frac{\left(h_{P}^{n}-h_{P}^{n-1}\right) \cdot d x \cdot d y}{d t} \\
f(t) & =Q_{\text {hydrograph }}(t), \text { or } Q^{n}
\end{aligned}
$$

The chosen method to solve those BC equations was Newton-Raphson method (Press et al. 1999), due to its stability and fast convergence. 


\section{Outlet Orifice and Weir}

The strategy for the outlet office and weir BCs is similar to that used for the inlet hydrograph BC. Depending on the desired BC, the characteristic equation is combined with Equations 5.10 or 5.5 (the orifice and the weir equations in Brater et.al., 1996) instead of the inlet hydrograph for the term $f(t)$ in the inlet BC.

$$
\begin{aligned}
& Q=A \cdot C_{d} \cdot \sqrt{2 g h_{o r}} \\
& Q=\frac{2}{3} \cdot L \cdot C_{d} \cdot \sqrt{2 g} \cdot\left(h_{\text {weir }}\right)^{3 / 2}
\end{aligned}
$$

where:

$$
\begin{aligned}
A & =\text { area of the orifice, } \\
L & =\text { length of the weir, } \\
C_{d} & =\text { discharge coefficient, } \\
h_{o r} & =\text { head in the orifice up from the orifice center, and } \\
h_{\text {weir }} & =\text { head in the weir up from the center of the orifice. }
\end{aligned}
$$

However, it was observed that when an inflow front arrives at the orifice, instabilities happen in the outlet $\mathrm{BC}$, resulting in high continuity errors. To avoid this problem, a step of $10 \mathrm{~cm}$ was considered from the bottom of the reservoir, so that when the flow starts at the $\mathrm{BC}$ it would not be with a shockwave front. Since the flow through the orifice starts in the moment it reaches the step level, $h_{o r}$ has its zero level in the center of the orifice, which means that the orifice was considered demoted until its center coincides with the top of the $10 \mathrm{~cm}$ step, as is shown in Figure 5.3.

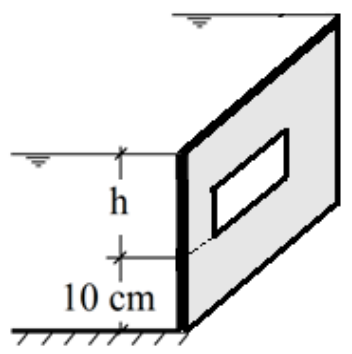

Figure 5.3 Typical $90^{\circ}$ orifice cell; the orifice is always considered to have a low height.

\subsubsection{Graphical User Interface}

Intended to be easy to use by engineers with practical knowledge in stormwater hydraulics but, not necessarily, of CFD, only the basic features 
used for a detention pond analysis were included, reducing the amount of input data and the degree of knowledge required to run the model. Given that the geometry and hydrograph data is known, the time necessary to enter the data from scratch to the start of the analysis of a detention pond with regular shape and little complexity is a few minutes, with the user inputting the inlet hydrograph, the height of the orifice, the pond's geometry (drawing it with simple clicks of the mouse), simulation time and the output file parameters, such as the time between two recorded results.

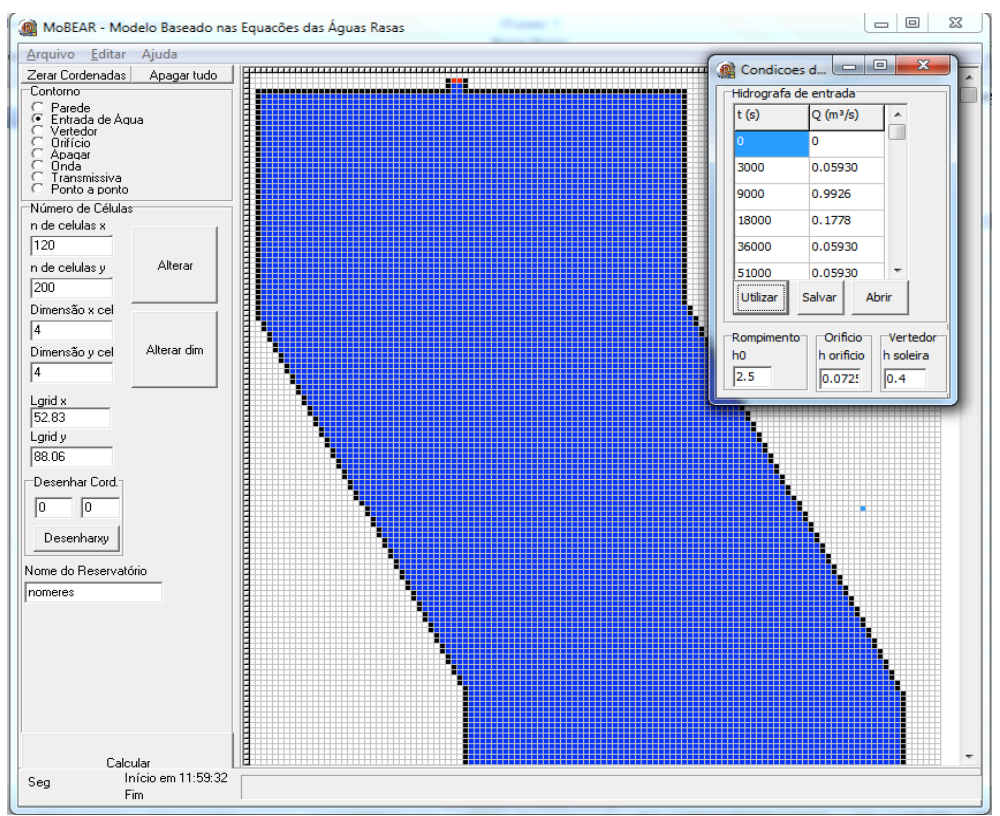

Figure 5.4 Example of the proposed model graphical user interface.

The post-processor developed was also focused on ease of use. Since it is integrated with GNUPlot, it can generate colored and isoline maps, hydrographs and 3-D surfaces with just one click of the mouse. It also organizes several different ponds that are part of the same system. A screen of the pre-processor interface (in Portuguese) is shown in Figure 5.4.

In this screen are shown the physical data input modules. The boundary conditions are drawn with the mouse and are listed in the checkbox on the top left side of the screen. The user needs only to select the desired BC and click on the checkered screen so that the model links each selected cell to the previous one with a straight line and fills the cells with a letter and a color representing the chosen $\mathrm{BC}$. 
After drawing the pond geometry, data such as the height of the orifice and the inlet hydrograph should be typed in the small dialog on the right side. When all the data is entered in the model, the user saves the input so that the program will analyze the geometry and automatically fill the interior part of the pond with blue. Note that the user can also draw obstacles within the pond, for example a rectangle, and the model will understand that the inside of this rectangle is dry, and so not to be considered as part of the solution domain.

\subsection{Model Testing and Results Analysis}

In order to test and compare the proposed model with the reservoir routing approach, two hypothetical ponds were analyzed. The first one has its geometry and inlet hydrograph based on an example proposed by Peine and Akan (2001) and the second has its geometry based on the Cambuci detention pond in São Paulo, Brazil. Since this is a comparative study, the inlet hydrographs are simplified, with just a few time and discharge points, representing a single hypothetical rain event for each.

For the operational analysis of the pond, simplified hypothetical criteria based only in the velocity fields were adopted in order to define what would be considered to be an operational issue. This is needed in order to illustrate how the post processor of a hydraulic model coupled with a proper sedimentation model would work. If the maximum velocity along the entire simulation in a particular cell exceeded $2 \mathrm{~m} / \mathrm{s}$, it was considered that scour would occur in this cell. Likewise, if the average velocity in a cell did not exceed $5 \mathrm{~mm} / \mathrm{s}$, it was considered that sediments would accumulate in that cell.

\subsubsection{Example 1 Paine and Akan (2001)}

This pond has a relatively simple geometry, as shown in Figure 5.5, with one inlet and one outlet structure. Its area is $2738 \mathrm{~m}^{2}$ and the peak inflow value is $0.9926 \mathrm{~m}^{3} / \mathrm{s}$, reached at $9000 \mathrm{~s}$. The pond was discretized with 55650 cells with a simulated time of $60000 \mathrm{~s}$. The outlet hydrographs for the SWE model and for the reservoir routing approach are shown in Figure 5.6.

In Figure 5.6 the shapes of the hydrographs and peak outflows for both methods were very similar. The peak outflow was $0.303 \mathrm{~m}^{3} / \mathrm{s}$ for the SWE model and $0.298 \mathrm{~m}^{3} / \mathrm{s}$ for the reservoir routing approach, a $1.5 \%$ difference. The late start of the SWE model's outflow hydrograph is due to the $10 \mathrm{~cm}$ head accumulation to start the orifice $\mathrm{BC}$ calculation, described earlier, and to the time the water takes to reach the outlet. The continuity error for this 
example was $5.7 \%$. Figure 5.7 shows a $3 \mathrm{D}$ picture of the free surface zoomed in as the outlet was generated for the instant $t=9000$. In this picture, there is a drop in the piezometric head right before the orifice. The average depth on the entire reservoir in this instant was $0.591 \mathrm{~m}$; the depth at the orifice region was $0.399 \mathrm{~m}$, which shows that of $0.192 \mathrm{~m}$ was converted into velocity head.

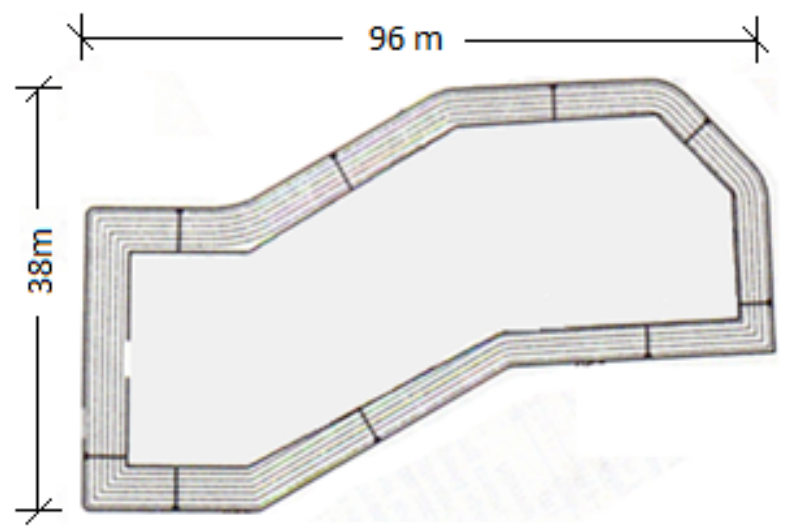

Figure 5.5 Paine and Akan (2001) detention pond.

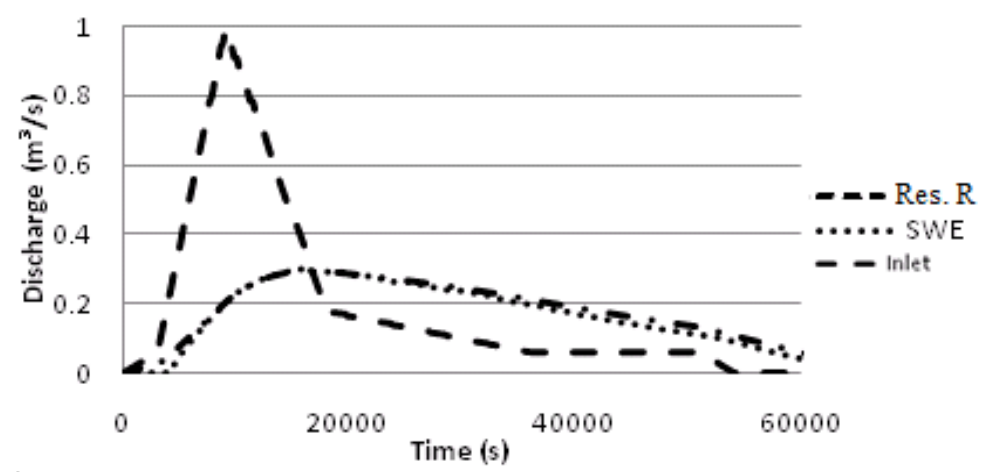

Figure 5.6 Hydrographs for example 1.

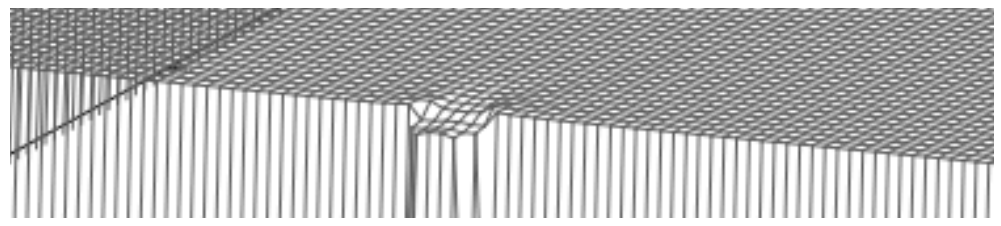

Figure 5.7 Representation out of scale of 3-D surface zoomed in the outlet orifice for example 1. 
In order to evaluate the zones where scour or sediment deposition would tend to occur, according to the simplified criteria outlined earlier, a set of velocity maps was generated. Figure 5.8 shows a map of maximum velocities observed in each cell of the domain along the entire simulation. It shows that no scour will occur in the reservoir for this rain event.

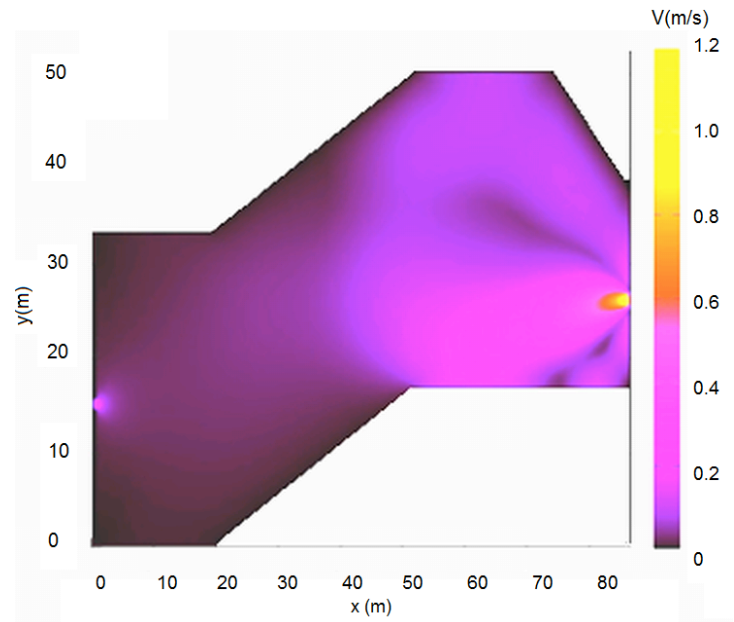

Figure 5.8 Map of maximum velocities reached in the simulation time for example 1.

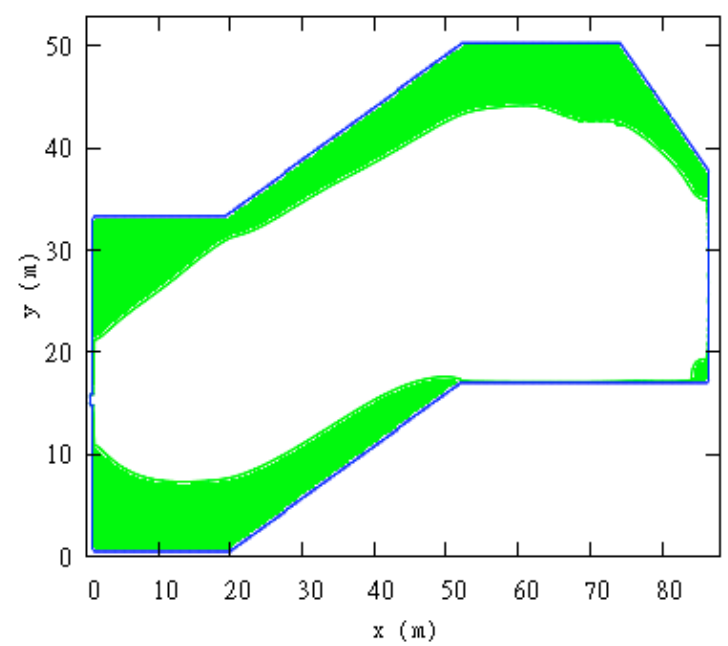

Figure 5.9 Zone more prone to sediment deposition according with simplified criteria for example 1.

The low average velocity map is presented in Figure 5.9. The intention of this map is to evaluate where sediment deposition may be more prone to 
occur. According to the simplified criteria mentioned in the methodology, the zones more prone to deposition are the closer to most of the borders. This zone corresponds to $28.6 \%$ of the pond area.

\subsubsection{Example 2 Simplified Version of Cambuci Detention Pond, São Paulo, Brazil}

The second comparison was performed with a detention pond similar in geometry to the Cambuci detention pond in São Paulo, Brazil. Its geometry is more complex than that of the previous example, as can be seen in Figure 5.10. Its area is $45977 \mathrm{~m}^{2}$ and the assumed inflow hydrograph has a peak of $40 \mathrm{~m}^{3} / \mathrm{s}$, reached in $9000 \mathrm{~s}$. The pond was discretized with 21838 cells and the simulated time was $40000 \mathrm{~s}$. The inlet and outlet hydrographs are shown in Figure 5.11.

As shown in Figure 5.11, the shapes of the hydrographs and peak outflows for both methods are different, especially in the recession hydrograph. The proposed model's recession hydrograph appears to be more accurate, since the decrease of the water level is supposed to be a power law because the head in the orifice is decreasing with the water level. The peak outflow was $20.09 \mathrm{~m}^{3} / \mathrm{s}$ for the model and $18.94 \mathrm{~m}^{3} / \mathrm{s}$ for the reservoir routing approach, which corresponds to a difference of $6.3 \%$.

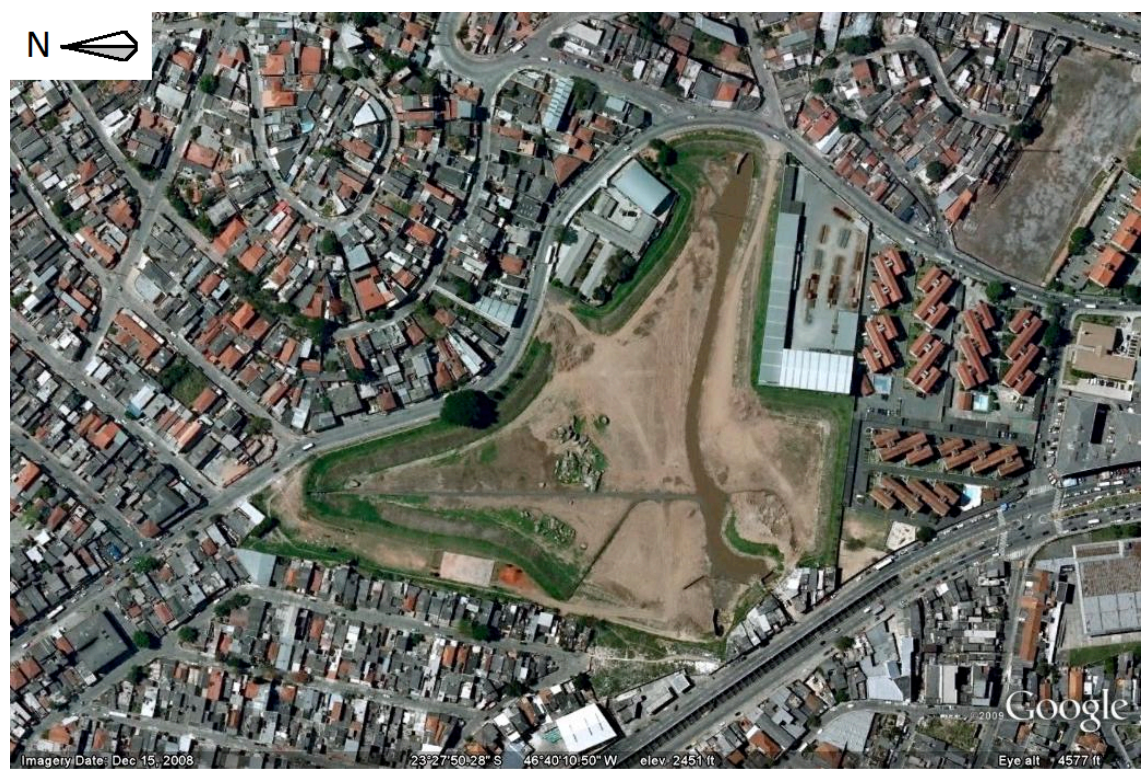

Figure 5.10 Aerial view of the Cambuci detention pond. 


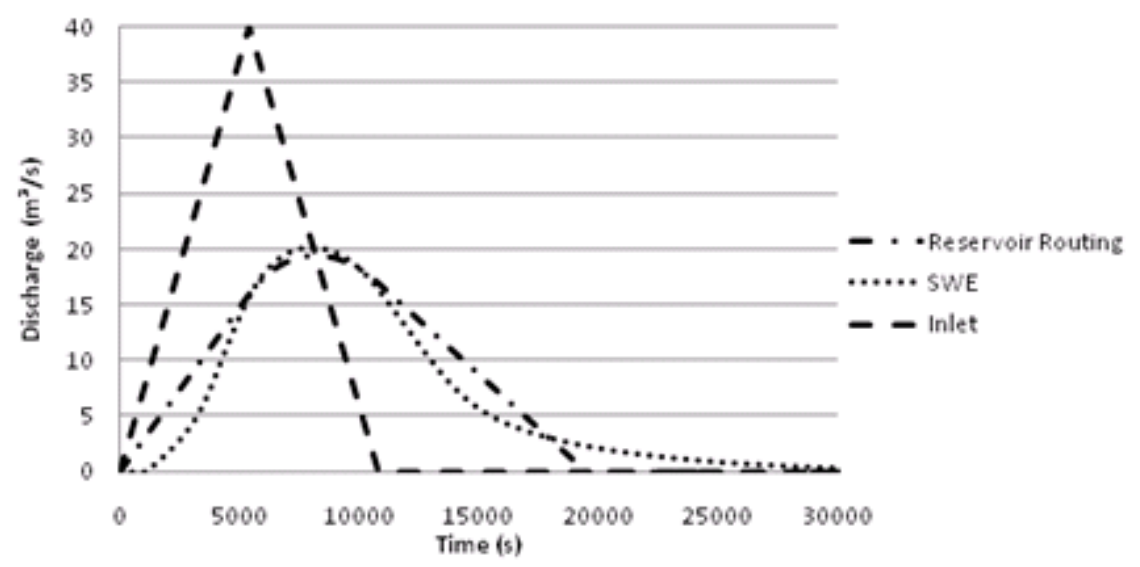

Figure 5.11 Hydrographs for example 2.

As in the last example, the late start of the proposed model's hydrograph is due to the $10 \mathrm{~cm}$ step to stabilize the orifice BC and to the time the water takes to reach the outlet. The continuity error for this simulation was $1.2 \%$. Figure 5.12 shows two cross-sectional views of the water profile for the instant $t=2000$.

In Figure 5.12, one notices a decrease in the piezometric head right before the orifice, as in the previous example. While the average depth on the entire reservoir in this instant was $0.478 \mathrm{~m}$, the depth (piezometric head) before the orifice was $0.054 \mathrm{~m}$, which indicates that $0.424 \mathrm{~m}$ of the piezometric head was converted into velocity head.

Figure 5.13 shows the map of the maximum velocities observed in each cell of the domain along the entire simulation. It shows that for this rain event, according to the simplified hypothetical criteria, scour will probably occur in the inlet of the reservoir. Also according to the simplified criteria, scour may occur close to the outlet.

From Figures 5.14 and 5.15 an average velocities map and some gross approximation for deposition zones on the right and on the left sides of the pond can be observed. Those zones correspond to $14.6 \%$ of the area of the pond. However, due to the $90^{\circ}$ edge approximation for non perpendicular sides (discretization limitations), this value was overestimated. 


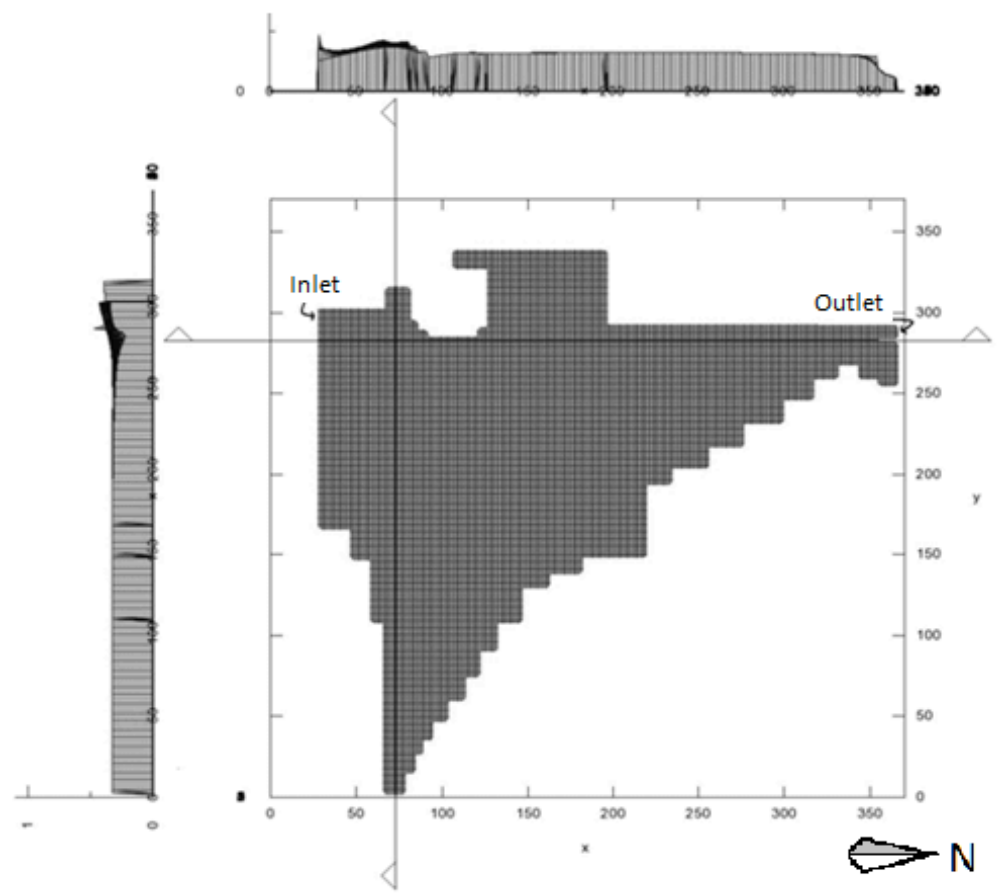

Figure 5.12 3-D surface and views from the Cambuci detention pond at a time $t=2000$.

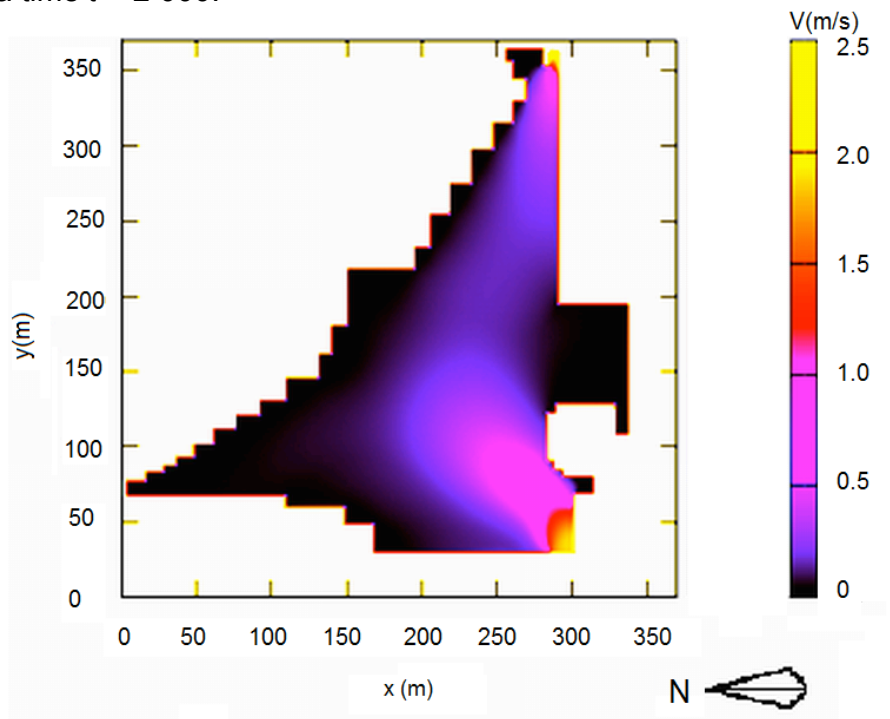

Figure 5.13 Map of maximum velocities reached in the simulation time for example 2. 


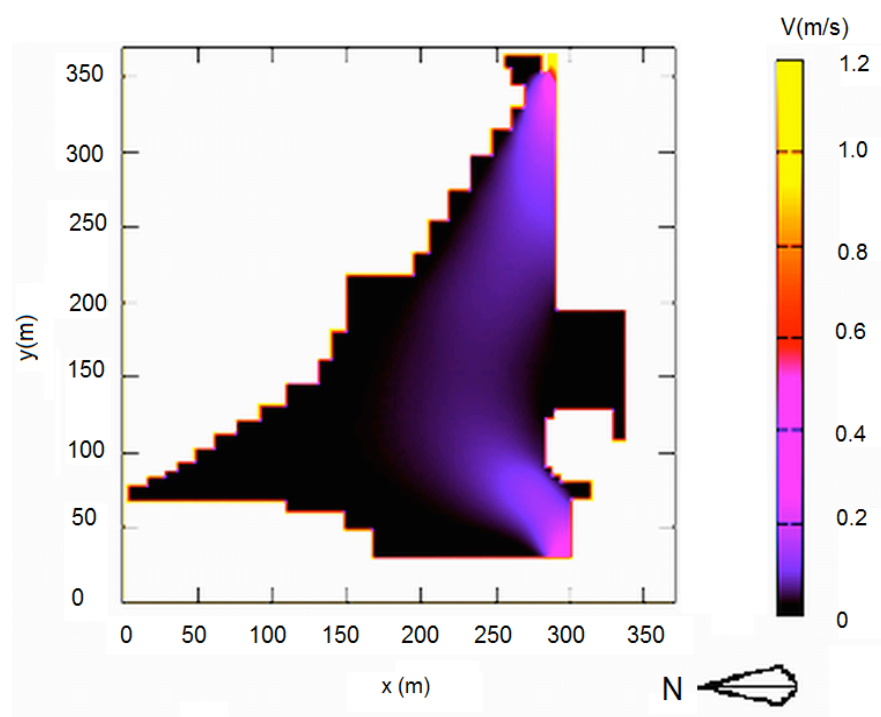

Figure 5.14 Map of average velocities reached in the simulation time for example 2.

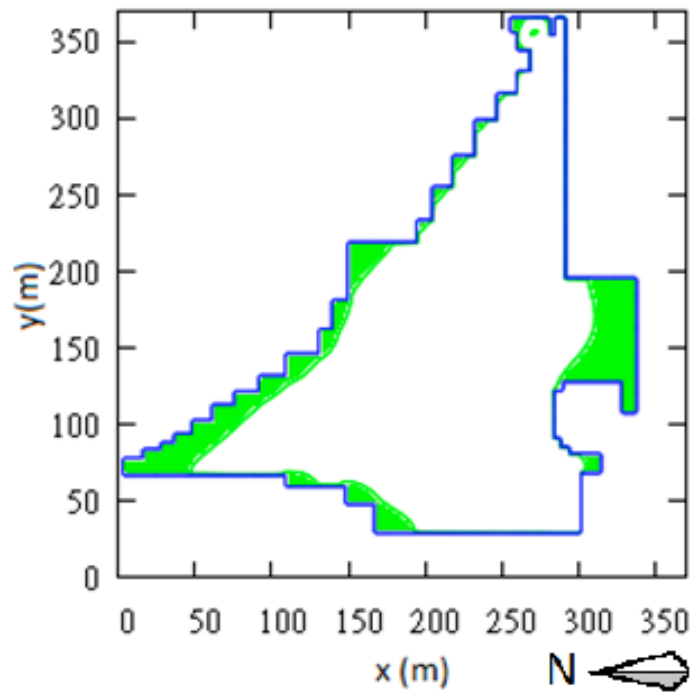

Figure 5.15 Zone more prone to sediment deposition according with simplified criteria for example 2.

\subsection{Final Discussion and Future Work}

For both detention ponds considered in this study, there was very good agreement between predicted outflow hydrographs by both the reservoir 
routing approach and the proposed model. Those results indicate the validity of the proposed model in calculating peak outflows for the given conditions.

However, the reservoir routing approach is unable to provide any information about operational issues that may arise if velocities within the pond are not adequate. This kind of flow information can be of great importance depending on the lining and of the maintenance conditions of the pond. The proposed model is able to provide such information, and is a useful tool for an initial screening of operational issues on detention reservoirs.

Because of its user friendly interface, the proposed model has the potential to be very useful to practitioners, after proper development and calibration phases. Unlike typical CFD packages, because there's great emphasis in making a simple user interface directed to the typical BCs encountered in detention ponds, users would be able to apply the model with much less training.

In Paine and Akan's example (example 1), there was very good agreement between the outlet hydrographs predicted by the reservoir routing approach and the proposed model. However, from the Cambuci based pond (example 2), there was some discrepancy on the shape of the recession hydrograph. The authors anticipate that the shape yielded by the proposed model is closer to the expected one, showing a power law decrease due to the decrease of the head in the orifice. The shape of this part of the hydrograph could be important for control of the drainage channels downstream from the pond. Wrong recession hydrographs for several detention ponds in the same drainage system can result in modeling errors that are avoidable.

Future developments of the proposed CFD model would need to incorporate source terms so that the model would be able simulate a pond with nonflat bottom topography and with shear forces. Nonlinear numerical schemes for intercell flux calculation to avoid numerical diffusion and oscillations shall also be made use of. Finally, and perhaps most importantly, the criterion for operational issues also would need to be perfected with the implementation of a proper sedimentation model, such as the ones presented in $\mathrm{Wu}$ (2009). The computational code should also be reviewed in order to make it more efficient. With those changes implemented, calibration of the model would be required before a broader application of the model.

\section{Acknowledgment}

The authors would like to acknowledge the support of the Universidade de Brasília, where the model was developed as the first author's final capstone project. 


\section{References}

Brater, E., King, H., Lindell, J. and Wei, C. (1996). Handbook of Hydraulics, McGrawHill Professional, 7 ed, 640 p.

Cunge, J. A., Holly, F. and Verwey, A. (1980). Practical Aspects of Computational River Hydraulics. Pitman Publishing Ltd., London. United kingdom, 420 p.

Miyaoka and Kawahara (2008). "Optimal control of drainage basin considering moving boundary”, International Journal of Computational Fluid Dynamics Vol. 22, No. 10, December 2008, 677-686.

Paine, J. N. and Akan, A. O. (2001). "Design of Detention Systems" in Stormwater Collection Systems Design Handbook. Org. Mays, L. W., McGraw-Hill Professional; 1 edition, $1008 \mathrm{p}$.

Press, W. H., Flannery, B. P., Teukolsky, S. A. and Vetterling W. T. (1999). Numerical Recipes in Pascal Cambridge University Press.

Qiu and Fang (2009). "Challenges on Three Dimensional Simulations of Free Surface Flow”, Proceeds from World Environmental and Water Resources Congress 2009: Great River: ASCE, Page 2747.

Sturm, T. W. (2001). Open Channel Hydraulics McGraw-Hill, New York, 512 p.

Toro, E. F. (2001). Shock-Capturing Methods for Free-Surface Shallow Flows. Wiley. $309 \mathrm{p}$.

USEPA (2009). Storm Water Management Model - SWMM, available at http://www.epa.gov/ednnrmrl/models/swmm/.

Vasconcelos, J. G. (2007). Métodos Numéricos Aplicados a Escoamentos Hidráulicos. Unpublished. Jul. 2007.

Williams, T. and Kelley, C. GNUPlot. (2004). Available at http://www.gnuplot.info/. Wu (2009), Computational River Dynamics, T\&F Books UK, 1 Edition, 494 p. 